\title{
Diagnostic and prognostic significance of cardiovascular magnetic resonance native myocardial T1 mapping in patients with pulmonary hypertension
}

Laura C. Saunders ${ }^{1}$, Chris S. Johns ${ }^{1}$, Neil J. Stewart ${ }^{1,2}$, Charlotte J. E. Oram ${ }^{1}$, David A. Capener ${ }^{1}$, Valentina O. Puntmann ${ }^{3}$, Charlie A. Elliot ${ }^{4}$, Robin C. Condliffe ${ }^{4}$, David G. Kiely ${ }^{4,5}$, Martin J. Graves ${ }^{6}$, Jim M. Wild ${ }^{1,5^{*}}$ and Andy J. Swift ${ }^{1,5}$

\begin{abstract}
Background: Native T1 may be a sensitive, contrast-free, non-invasive cardiovascular magnetic resonance (CMR) marker of myocardial tissue changes in patients with pulmonary artery hypertension. However, the diagnostic and prognostic value of native T1 mapping in this patient group has not been fully explored. The aim of this work was to determine whether elevation of native $\mathrm{T} 1$ in myocardial tissue in pulmonary hypertension: (a) varies according to pulmonary hypertension subtype; (b) has prognostic value and (c) is associated with ventricular function and interaction.

Methods: Data were retrospectively collected from a total of 490 consecutive patients during their clinical 1.5 T CMR assessment at a pulmonary hypertension referral centre in 2015. Three hundred sixty-nine patients had pulmonary hypertension [58 \pm 15 years; $66 \%$ female], an additional 39 had pulmonary hypertension due to left heart disease [68 \pm 13 years; $60 \%$ female], 82 patients did not have pulmonary hypertension [ $55 \pm 18 ; 68 \%$ female]. Twenty five healthy subjects were also recruited [58 \pm 4 years); $51 \%$ female]. T1 mapping was performed with a MOdified Look-Locker Inversion Recovery (MOLLI) sequence. T1 prognostic value in patients with pulmonary arterial hypertension was assessed using multivariate Cox proportional hazards regression analysis.

Results: Patients with pulmonary artery hypertension had elevated T1 in the right ventricular (RV) insertion point (pulmonary hypertension patients: $\mathrm{T} 1=1060 \pm 90 \mathrm{~ms}$; No pulmonary hypertension patients: $\mathrm{T} 1=1020 \pm 80 \mathrm{~ms} p<0.001$; healthy subjects T1 $=940 \pm 50 \mathrm{~ms} p<0.001$ ) with no significant difference between the major pulmonary hypertension subtypes. The RV insertion point was the most successful T1 region for discriminating patients with pulmonary hypertension from healthy subjects (area under the curve $=0.863$ ) however it could not accurately discriminate between patients with and without pulmonary hypertension (area under the curve $=0.654$ ). T1 metrics did not contribute to prediction of overall mortality (septal: $p=0.552$; RV insertion point: $p=0.688$; left ventricular free wall: $p=0.258$ ). Systolic interventricular septal angle was a significant predictor of $\mathrm{T} 1$ in patients with pulmonary hypertension $(p<0.001)$.

Conclusions: Elevated myocardial native T1 was found to a similar extent in pulmonary hypertension patient subgroups and is independently associated with increased interventricular septal angle. Native T1 mapping may not be of additive value in the diagnostic or prognostic evaluation of patients with pulmonary artery hypertension.
\end{abstract}

Keywords: Pulmonary hypertension, T1 mapping, Cardiovascular magnetic resonance, MOLLI

\footnotetext{
* Correspondence: j.m.wild@sheffield.ac.uk

${ }^{1}$ POLARIS, Academic Radiology, Department of Infection, Immunity and

Cardiovascular Disease, University of Sheffield, Sheffield, UK

${ }^{5}$ INSIGNEO, Institute for in-silico medicine, Sheffield, UK

Full list of author information is available at the end of the article
}

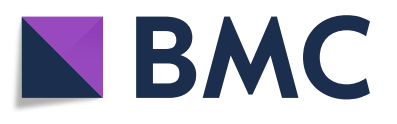

(c) The Author(s). 2018 Open Access This article is distributed under the terms of the Creative Commons Attribution 4.0 International License (http://creativecommons.org/licenses/by/4.0/), which permits unrestricted use, distribution, and reproduction in any medium, provided you give appropriate credit to the original author(s) and the source, provide a link to the Creative Commons license, and indicate if changes were made. The Creative Commons Public Domain Dedication waiver (http://creativecommons.org/publicdomain/zero/1.0/) applies to the data made available in this article, unless otherwise stated. 


\section{Background}

Myocardial tissue changes have been visualised in patients with pulmonary hypertension using cardiovascular magnetic resonance (CMR) T1 mapping, T2 mapping and late gadolinium enhancement (LGE) CMR. Native myocardial changes measured using native T1 mapping have been shown across a range of cardiac diseases in clinical subjects $[1,2]$. It has been shown that in patients with pulmonary artery hypertension (PAH) the right ventricular (RV) insertion point T1 is elevated. Several studies also found elevated $\mathrm{T} 1$ in the interventricular septum and, in one case, the left ventricular (LV) free wall [3-7].

Patients with pulmonary hypertension have been shown to have worse prognosis in the presence of myocardial changes visualized by LGE at the RV insertion point [8] and in the septal myocardium [9], although the independent prognostic significance of the myocardial tissue changes in pulmonary hypertension remains unclear [10]. Elevated native T1 at the insertion point occurs in a similar location and pattern to LGE. The co-occurrence of LGE and elevated T1 implies that they may have common mechanistic causes. However, studies in both patients and animal models have reported higher native $\mathrm{T} 1$ in the $\mathrm{RV}$ insertion points than in controls, despite no LGE being seen in this region, which indicates that native $\mathrm{T} 1$ may be a more sensitive marker of myocardial changes than the presence of LGE $[3,11]$. Therefore, T1 mapping may provide a contrast-free and sensitive marker of myocardial tissue changes in pulmonary artery hypertension, and there is reason to suspect that $\mathrm{T} 1$ changes may have prognostic relevance.

Pulmonary hypertension is a heterogeneous condition with 5 diagnostic subcategories: PAH; pulmonary hypertension due to left heart disease pulmonary hypertension due to lung disease and/or hypoxia; chronic thromboembolic pulmonary hypertension (CTEPH) and pulmonary hypertension with unclear or multifactorial causes. To date T1 mapping studies have been performed in relatively small populations, with only one report wherein diagnostic subcategories were considered separately [4] and therefore it is unclear whether myocardial $\mathrm{T} 1$ changes in patients with pulmonary hypertension are homogeneous across patient subtypes.

RV remodelling and failure occur as a result of prolonged elevations of RV afterload. Elevated mean pulmonary artery pressure (PAP) and pulmonary vascular resistance (PVR) are characteristic features of the condition, however it is the failure of the RV that is the key determinant of adverse outcome [9, 12-16]. Early markers of adverse cardiac remodelling may be able to guide treatment strategies, predicting therapy response and failure. It is unclear whether elevated myocardial T1 is associated with prognostic features in pulmonary hypertension, and whether it can provide additive information to patient outcome.

In this work, we sought to determine whether elevation of the native T1 in myocardial tissue in pulmonary hypertension: (a) varies according to pulmonary hypertension subtype; (b) is related to adverse outcome independent of RV size and function, and (c) is associated with biventricular remodeling and function.

\section{Methods \\ Subjects}

Approval for analysis of imaging data was granted by the local research ethics committee and consent was waived for this retrospective database study. Consecutive patients with suspected pulmonary hypertension underwent CMR including a modified Look-Locker inversion recovery (MOLLI) sequence as part of their clinical assessment at a tertiary pulmonary hypertension referral centre between 01.01.2015 and 31.12.2015. Exclusion criteria were applied to all subjects according to standard criteria for undergoing CMR. A total of 490 patients were identified. Three hundred sixty-nine patients had a diagnosis of pulmonary hypertension without left heart disease as specified below, 39 patients were identified with pulmonary hypertension associated with left heart disease, and 82 did not have a diagnosis of pulmonary hypertension - mean PAP $<25 \mathrm{mmHg}$ - and were used as a control group, see Fig. 1. Twenty five age matched healthy subjects with no current cardiac or respiratory symptoms and no history of cardiac disorders were also recruited as an additional control group. Patients with a mean $\mathrm{PAP} \geq 25 \mathrm{mmHg}$ and pulmonary capillary wedge pressure $(\mathrm{PCWP})>15 \mathrm{mmHg}$ were considered to have a diagnosis of pulmonary hypertension due to left heart disease, and patients with a mean PAP $<25 \mathrm{mmHg}$ were considered to have a diagnosis of no pulmonary hypertension. Subtypes of pulmonary hypertension were defined as previously described [17].

The patients with pulmonary hypertension included the following sub-group diagnoses: Group 1 pulmonary arterial hypertension; Idiopathic PAH, $n=83$; $\mathrm{PAH}$ heritable, $n=5$; $\mathrm{PAH}$ associated with connective tissue disease (PAH-CTD), $n=89$; $\mathrm{PAH}$ associated with congenital heart disease (CHD), $n=31$; PAH associated with portal hypertension, $n$ =9; $\mathrm{PAH}$ drug/toxin induced, $\mathrm{n}=3 ; \mathrm{PAH}$ other, $n=3$. Group 2 left heart disease, $n=39$ (note these patients were considered separately - see Fig. 1). Group $3 \mathrm{PH}$ associated with lung disease, $n=41$. Group 4, CTEPH $n=92$; group 5 , sarcoidosis, $n=10$; patients without diagnosis: 3 .

All patients with PAH $(n=223)$ were treated with PAH therapy. Patients with PAH were strictly defined for this study as PCWP $<16$ and no evidence of lung disease or left heart disease. Chronic thromboembolic pulmonary hypertension $(\mathrm{CTEPH})$ patients and some 


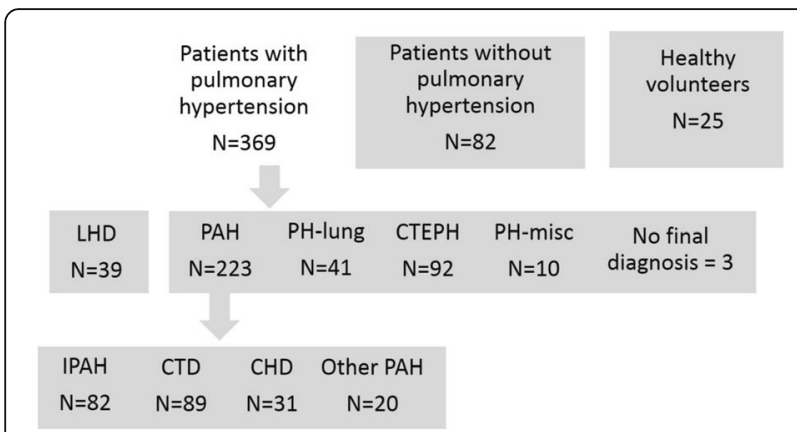

Fig. 1 Patient flowchart. Patients were grouped for statistical analysis as indicated. Patients with pulmonary hypertension were compared to two control groups. Patients with left heart disease (LHD), pulmonary arterial hypertension (PAH), PAH due to lung disease and/or hypoxia (PH-lung), chronic thromboembolic pulmonary hypertension (CTEPH) and pulmonary hypertension with unclear or multifactorial causes ( $\mathrm{PH}$-misc) were compared using ANOVA. Patients with idiopathic pulmonary arterial hypertension (IPAH), connective tissue disease (CTD), congenital heart disease $(\mathrm{CHD})$ and other types of PAH were compared using ANOVA. Patients with PAH were assessed for independent prognostic value of $\mathrm{T} 1$. Three patients had previous right heart catheter data showing mean PAP $>25 \mathrm{mmHg}$ but did not have a final diagnosis, and were included in analysis of pulmonary hypertension patients

aetiologies of Group 5 pulmonary hypertension were treated as appropriate. Patients with left heart disease were divided into two groups, firstly typical left heart disease $(n=35)$ and secondly, PAH with left heart disease comorbidity $(n=4)$. Patients in the PAH associated with lung disease were divided into severe PAH-lung disease $(n=13)$ and PAH with non-severe lung disease comorbidity $(n=28)$. A summary of all subject characteristics is shown in Table 1, and a detailed breakdown of the subgroups provided in Additional file 1: Table S1.

\section{Right heart catheterisation}

All patients underwent right heart catheterisation. Right heart catheterisation was performed by a using a balloon-tipped 7.5 Fr thermodilution catheter (BectonDickinson, Franklin Lakes, New Jersey, USA), typically via the internal jugular vein using a Swan-Ganz catheter. PVR index (PVRI) was defined as (mPAP-PCWP)/CI, where $\mathrm{CI}$ is cardiac index, measured by thermodilution technique. Patients with right heart catheterisation within 30 days of their CMR were included in analysis of associations between right heart catheterisation metrics and myocardial $\mathrm{T} 1$.

\section{CMR acquisition}

CMR was performed on a whole body $1.5 \mathrm{~T}$ scanner (HDx scanner, GE Healthcare, Waukesha, Wisconsin, USA), using an 8-channel cardiac coil. Subjects were scanned in the supine position with electrocardiogram (ECG) gating. Short axis cine images were acquired using a multi-slice balanced steady state free precession (bSSFP) sequence with: temporal phases per cardiac cycle: 20; field of view: $480 \mathrm{~mm}$; matrix: $256 \times 256$; bandwidth: $125 \mathrm{KHz} /$ pixel; TR: $3.7 \mathrm{~ms}$; TE: $1.6 \mathrm{~ms}$.

T1 mapping was performed using a 2D 3-3-5 MOLLI sequence in a single short axis slice [17]. A bSSFP acquisition was executed at each inversion time point with the following sequence parameters: Flip angle: $35^{\circ}$; image dimensions: $128 \times 128$; TR: $3.20 \mathrm{~ms}$; TE: $1.41 \mathrm{~ms}$; parallel imaging using sensitivity encoding with acceleration factor 2; FOV: $400 \mathrm{~mm}$; slice thickness: $5.1 \mathrm{~mm}$.

\section{CMR image analysis}

Cine CMR analysis was performed prospectively on a GE Advantage Workstation 4.1 by a radiographer with 10 years' experience, who was blinded to the patient's clinical data. Right and left endocardial and epicardial surfaces were manually traced on the short axis cine images to obtain the volumetric indices; RV end diastolic volume (RVEDV), RV end systolic volume (RVESV), LV end diastolic volume (LVEDV) and LV end systolic volume (LVESV). From the end diastolic and end systolic volumes, RV ejection fraction (RVEF) and LV ejection fraction (LVEF) were calculated. The interventricular septum was included in the LV mass. RV mass was estimated from the sum total of the myocardial slice volumes and an assumed myocardial density of $1.05 \mathrm{~g} / \mathrm{cm}^{3}$. Ventricular mass index was calculated as $\mathrm{VMI}=\mathrm{RV}$ mass / LV mass. CMR volume and mass measurements were indexed for body surface area where appropriate. Interventricular septal angle was measured by determining the angle between the mid-point of the interventricular septum and the $R V$ insertion points at RV end systole [18].

All MOLLI images were spatially registered to correct any respiratory or cardiac motion using a Matlab (Mathworks, Natick, Massachusetts, USA)-based non-rigid image registration algorithm [19], which was integrated into a Matlab voxelwise T1 mapping algorithm based on the work by Xue et al. [20]. Analysis of T1 maps was performed by a Cardiothoracic Radiologist with 7 years CMR experience, blinded to the patient's demographic and clinical data. Regions of interest were drawn on the interventricular septum, RV insertion points and LV free wall, see Fig. 2 [21]. A measure of $\Delta \mathrm{T} 1_{\mathrm{RS}}$ was used to assess elevation of the T1 in the RV insertion points, normalised to septal T1. Regions of interest were compared across pulmonary hypertension subtypes (see flowchart in Fig. 1). All T1 maps were analysed by both the same observer, and a second observer, who was a trained CMR researcher with 2 years of experience analysing T1 mapping images blinded to previous results and patient data for interobserver and intraobserver reproducibility. 
Table 1 Characteristics of subjects

\begin{tabular}{|c|c|c|c|c|}
\hline \multirow[t]{2}{*}{ N } & Patients: PAH & Group 3 PH LHD & Patients: no PAH & Healthy Subjects \\
\hline & 369 & 39 & 82 & 24 \\
\hline \multicolumn{5}{|l|}{ Demographics and characteristics } \\
\hline Age (years) & $58.2 \pm 15.0$ & $68.1 \pm 12.7^{* *}$ & $55.4 \pm 18.3$ & $58.1 \pm 3.88$ \\
\hline Sex (female \%) & 66.0 & 59.5 & 67.1 & 50.9 \\
\hline Subgroup diagnoses (n) & & N/A & N/A & - \\
\hline Group 1 PAH & $n=223$ & & & \\
\hline PH-Lung & $n=41$ & & & \\
\hline CTEPH & $n=92$ & & & \\
\hline PH-MISC & $n=10$ & & & \\
\hline $\mathrm{BSA}\left(\mathrm{m}^{2}\right)$ & $1.81 \pm 250$ & $1.89 \pm 0.26$ & $1.85 \pm 0.25$ & \\
\hline \multicolumn{5}{|l|}{ CMR } \\
\hline Heart Rate (bpm) & $72.17 \pm 14.4$ & $69.81 \pm 3.8$ & $67.9 \pm 10.3^{* *}$ & \\
\hline CMR at incident vs prevalent time (incident \%) & $N=165(45 \%)$ & $N=31(80 \%)$ & $N=82(100 \%)$ & \\
\hline RV insertion $\mathrm{T} 1$ (ms) & $1065 \pm 86$ & $1074 \pm 10$ & $1017 \pm 69$ & $943 \pm 52^{* *}$ \\
\hline Septal T1 (ms) & $975 \pm 67$ & $986 \pm 73$ & $976 \pm 75$ & $940 \pm 56^{*}$ \\
\hline LV free wall T1 (ms) & $965 \pm 68$ & $982 \pm 81$ & $961 \pm 66$ & $913 \pm 55^{* *}$ \\
\hline RVEDV Index $\left(\mathrm{ml} / \mathrm{m}^{2}\right)$ & $87.2 \pm 34.1$ & $81.9 \pm 31.7$ & $68.0 \pm 20.4^{* *}$ & \\
\hline RVESV Index $\left(\mathrm{ml} / \mathrm{m}^{2}\right)$ & $51.7 \pm 29.0$ & $45.4 \pm 23.1$ & $31.1 \pm 11.6^{* *}$ & \\
\hline RVSV Index (\%) & $35.6 \pm 14.2$ & $36.5 \pm 12.1$ & $36.9 \pm 12.9$ & \\
\hline RVEF (\%) & $43.1 \pm 13.6$ & $46.8 \pm 11.2$ & $54.4 \pm 10.0^{* *}$ & \\
\hline LVEDV Index $\left(\mathrm{ml} / \mathrm{m}^{2}\right.$ & $58.5 \pm 16.1$ & $68.9 \pm 21.2^{* *}$ & $66.6 \pm 16.1^{* *}$ & \\
\hline LVESV Index $\mathrm{ml} / \mathrm{m}^{2}$ ) & $19.2 \pm 8.2$ & $26.1 \pm 16.6^{* *}$ & $21.3 \pm 8.2^{*}$ & \\
\hline LVSV Index (\%) & $39.2 \pm 11.9$ & $42.8 \pm 13.4$ & $45.3 \pm 10.8^{* *}$ & \\
\hline LVEF (\%) & $67.3 \pm 9.7$ & $63.8 \pm 14.5$ & $68.3 \pm 7.6$ & \\
\hline Systolic septal angle $\left(^{\circ}\right)$ & $165.5 \pm 23.9$ & $145.9 \pm 15.5$ & $150.5 \pm 108.4^{*}$ & \\
\hline Diastolic septal angle $\left(^{\circ}\right)$ & $143.34 \pm 11.0$ & $138.3 \pm 10.3^{* *}$ & $134.9 \pm 7.9^{*}$ & \\
\hline RV mass index $\left(\mathrm{g} / \mathrm{m}^{2}\right)$ & $21.9 \pm 12.5$ & $14.4 \pm 5.5^{* *}$ & $11.9 \pm 5.8^{*}$ & \\
\hline LV mass index $\left(\mathrm{g} / \mathrm{m}^{2}\right)$ & $48.0 \pm 12.3$ & $57.2 \pm 21.1^{* *}$ & $47.7 \pm 10.7$ & \\
\hline VMI (ratio) & $0.47 \pm 0.27$ & $0.28 \pm 0.13^{* *}$ & $0.26 \pm 0.18^{*}$ & \\
\hline
\end{tabular}

WHO world health organisation, $P A H$ ipulmonary arterial hypertension, CTEPH chronic thromboembolic pulmonary hypertension, RVEDV right ventricular end diastolic volume, RVESV right ventricular end systolic volume, RVSV right ventricular stroke volume, RVEF right ventricular ejection fraction, VMI ventricular mass index, LVEDV left ventricular end diastolic volume, LVESV left ventricular end systolic volume, LVSV left ventricular stroke volume, LVEF left ventricular ejection fraction, $V M I$ ventricular mass index, CMR cardiovascular magnetic resonance

$P$ values indicate comparisons between patients with $\mathrm{PH}$ and the other two groups. ${ }^{*}$ signifies a $p$ value $<0.05,{ }^{*}$ signifies a $p$ value $<0.025$

\section{Follow up period}

Patients with PAH were followed up with a census date of 30.01.2018 with a mean follow-up period of $27 \pm$ 8 months and a median follow-up period of 30 months from CMR to death or census.

\section{Statistical analysis}

Statistical analysis was performed and presented using SPSS 21 (SPSS, Statistical Package for the Social Sciences, International Business Machines, Inc., Armonk, New York, USA). Data are presented as mean \pm standard deviation. Group comparisons were made using two-tailed ANOVA; post-hoc Bonferroni was used for multiple group corrections. Pearson's correlation coefficient was used to assess the strength of correlations. Receiver operating characteristic (ROC) analysis was employed to assess the diagnostic accuracy of $\mathrm{T} 1$ metrics with area under the curve. Intra and interobsever variability was assessed using the intraclass correlation coefficient (ICC); absolute values and 95\% confidence intervals. To control for the heterogeneity of aetiology and therapy differences between the different subtypes of pulmonary hypertension, we elected to study the prognostic value of $\mathrm{T} 1$ metrics in patients with $\mathrm{PAH}$ alone. This controls for differences in outcome caused by factors such as 


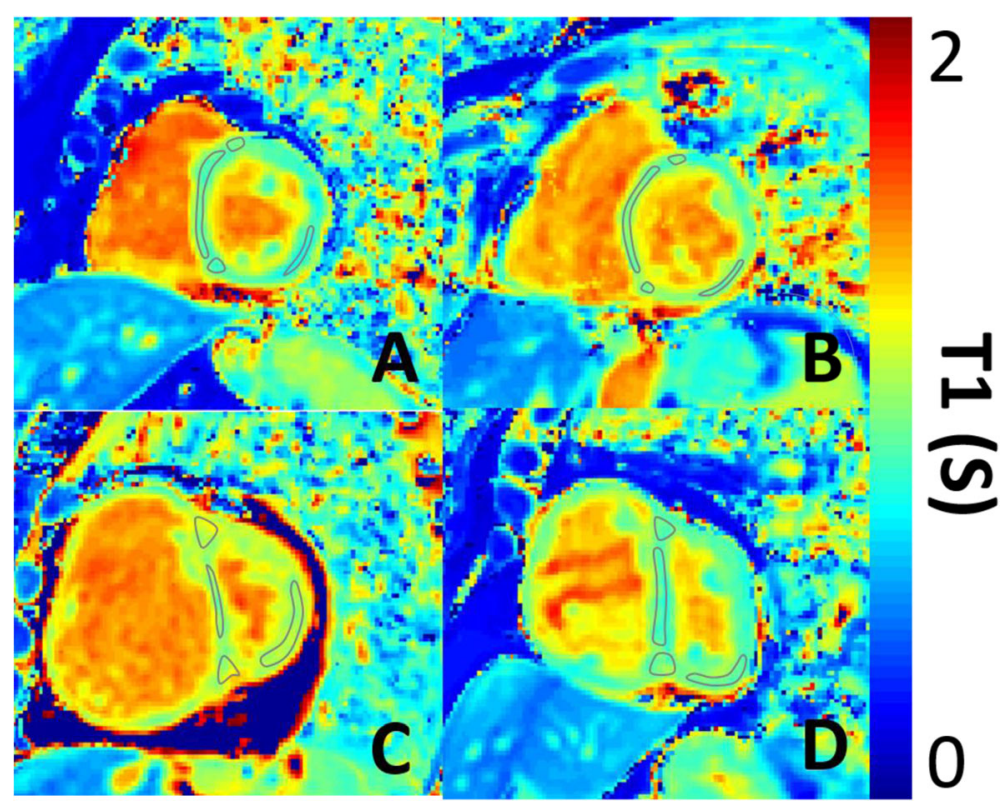

Fig. 2 Representative T1 maps. Representative T1 maps in short axis geometry of a) a healthy subject, b) a patient without pulmonary artery hypertension, $\mathbf{c}$ ) a patient with idiopathic PAH and $\mathbf{d}$ ) a patient with left heart diseaes. Demonstrative regions of interest are places on the RV insertion points, interventricular septum and LV free wall

severe lung disease in respiratory patients and chronic thromboembolic disease in patients undergoing pulmonary endarterectomy. Log-log plots were produced for T1 metrics to assess proportional hazards, with variables dichotomised by median values, and prognostic value was assessed using univariate Cox proportional hazards regression analysis. Variable scaling (Z-score) was performed to allow direct comparison of hazard ratios of all continuous variables by subtracting the mean from individual values and dividing by the standard deviation of the variable. Groups were compared using the log-rank (Mantel-Cox) test. Unless otherwise stated, a value of $p<0.05$ was considered statistically significant.

\section{Results}

Characteristics of the study populations are given in Table 1. Additional data on characteristics of PAH subtypes are given in Additional file 1: Tables S1 and S2. Patients with PAH were not significantly different in age or sex than patients without PAH or healthy subjects. Figure 1 illustrates the classification of the patients included in the study.

\section{Native myocardial T1 mapping}

In patients with $\mathrm{PAH}, \mathrm{T} 1$ was significantly elevated at the RV insertion points when compared to healthy subjects and patients without PAH (patients with pulmonary hypertension, $\mathrm{T} 1=1065 \pm 86 \mathrm{~ms}$; healthy subjects, T1 $=943 \pm 52 \mathrm{~ms}, p<0.001$; patients $\mathrm{PAH} \mathrm{T} 1=$ $1017 \pm 69 \mathrm{~ms}, \mathrm{p}<0.001)$. Patients with PAH also had elevated $\mathrm{T} 1$ in the $\mathrm{LV}$ free wall and septum when compared to healthy subjects but not patients PAH, (patients with PAH: LV free wall $\mathrm{T} 1=975 \pm 67 \mathrm{~ms}$, septal $\mathrm{T} 1=965 \pm$ $68 \mathrm{~ms}$; patients without PAH: LV free wall $\mathrm{T} 1=961 \pm$ $66 \mathrm{~ms}$, septal $\mathrm{T} 1=976 \pm 75 \mathrm{~ms}$; healthy subjects LV free wall $\mathrm{T} 1=913 \pm 55 \mathrm{~ms}, \mathrm{p}<0.001$, septal $\mathrm{T} 1=940 \pm$ $56 \mathrm{~ms}, p=0.011$ ). See Table 1 and Figs. 2, 3. Patients with PAH who were undergoing treatment did not have significantly different $\mathrm{T} 1$ to those who were untreated (septal: $p=0.182$; RV insertion point: $p=0.977$; LV free wall: $p=0.46$ ). The standard deviation of $\mathrm{T} 1 \mathrm{~s}$ within the RV insertion points was significantly higher in patients with PAH ( $\mathrm{SD}=62.2 \mathrm{~ms}$ ) compared to healthy subjects ( $\mathrm{SD}=36.5 \mathrm{~ms})$. The standard deviation of $\mathrm{T} 1 \mathrm{~s}$ within the LV free wall and septum were not significantly different in healthy subjects when compared to patients (septal: $54.4 \mathrm{~ms}$, LV free wall: $42.9 \mathrm{~ms}$ ) with PAH (septal: $54.6 \mathrm{~ms}$, LV free wall: $44.6 \mathrm{~ms}$ ).

\section{Diagnostic accuracy of myocardial T1}

The $\mathrm{T} 1$ at the RV insertion points was the most statistically significant measurement for discriminating between subjects and patients with $\mathrm{PAH}$, followed by $\Delta \mathrm{T} 1_{\mathrm{RS}}$. However, no T1 region was successful in discriminating between patients without PAH and patients with PAH, see Table 2. 


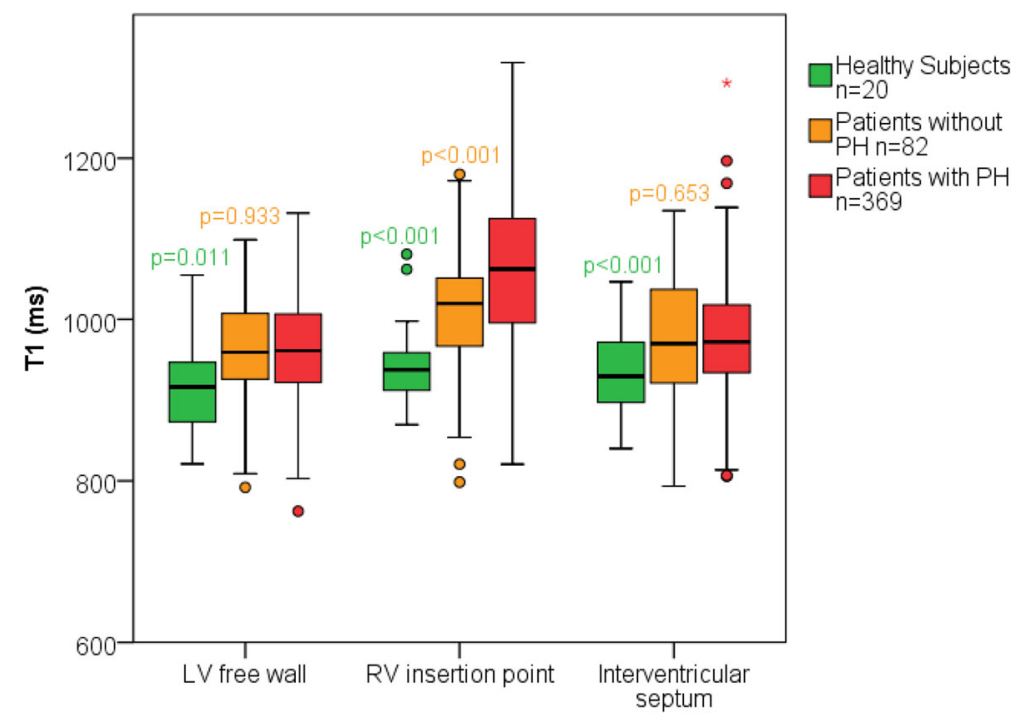

Fig. 3 Mean $\mathrm{T} 1$ in patients with pulmonary artery hypertension, patients without pulmonary artery hypertension and healthy subjects. Box plot of the mean T1 for septal, RV insertion point and LV free wall regions. $P$ values are given for ANOVA comparisons between patients with pulmonary artery hypertension and control groups above each respective control group. O Represents outliers * represents far outliers

T1 changes in pulmonary artery hypertension subtypes Comparisons were made between PAH subgroups and there were no significant differences between subgroups. For PAH patients, LV free wall T1 was significantly higher $(p=0.046)$ in patients with CTD than patients with CHD. There were no other significant differences between groups, see Fig. 4 .

\section{Relationship between $\mathrm{T} 1$ and other measures of cardiac structure and function}

Across the population of PAH patients scanned, the RV insertion point $\mathrm{T} 1$ had the strongest relationship with $\mathrm{RV}$ function. The mean insertion point $\mathrm{T} 1$ correlated significantly with RVEDV index, RVESV index and RVEF. Additionally, the RV insertion point T1 showed

Table 2 ROC curve analysis for distinguishing patients without $\mathrm{PH}$ and patients with $\mathrm{PH}$

\begin{tabular}{llll}
\hline & $\begin{array}{l}\text { Area under } \\
\text { the curve }\end{array}$ & $\begin{array}{l}\text { Lower 95\% } \\
\text { Confidence interval }\end{array}$ & $\begin{array}{l}\text { Upper 95\% } \\
\text { Confidence interval }\end{array}$ \\
\hline $\begin{array}{l}\text { RV mass } \\
\text { Index }\end{array}$ & 0.807 & 0.760 & 0.855 \\
$\begin{array}{l}\text { Systolic } \\
\text { septal angle }\end{array}$ & 0.850 & 0.809 & 0.892 \\
Septal T1 & 0.502 & 0.426 & 0.578 \\
RV insertion & 0.654 & 0.587 & 0.720 \\
point T1 & & & \\
$\begin{array}{l}\Delta \text { T1 }_{\text {RVIP-septum }} \\
\text { LV free wall }\end{array}$ & 0.675 & 0.612 & 0.738 \\
T1 & 0.512 & 0.442 & 0.582 \\
\hline Legend: $\Delta$ T1 $_{\text {RVIP-septum }}=$ RV insertion point T1 - septal T1 &
\end{tabular}

significant correlations with RV mass index, the VMI and the systolic and diastolic septal angle, see Table 3. Septal T1 and LV free wall T1 did not correlate with any of the imaging markers of RV function or LV function. However, diastolic septal angle and septal T1 showed a positive correlation $(R=0.152)$, see Table 3 .

A multivariate forward linear regression showed that RVESVI and systolic septal angle were significant independent predictors of RV insertion point $\mathrm{T} 1$ in patients with PAH.

In patients with left heart disease the RV insertion point $\mathrm{T} 1$, septal $\mathrm{T} 1$ and $\mathrm{LV}$ free wall $\mathrm{T} 1$ all showed significant correlations with cine CMR metrics of RV function. $\Delta \mathrm{T} 1_{\mathrm{RS}}$ correlated significantly with diastolic and systolic septal angle in patients with left heart disease, (see Table 4).

A total of 228/369 patients with PAH, 30/39 patients with PAH due to left heart disease and 29/82 patients without $\mathrm{PAH}$ also had right heart catheter within 1 month of CMR scanning (mean of $0 \pm 1$ days, range 15 days). Mean PAP for each patient group was as follows: patients with PAH: $44 \pm 13$; patients with $\mathrm{PAH}$ and left heart disease: $40 \pm 12$; patients without PAH $19 \pm 3$. Right heart catheter data is presented for patient subjects in Additional file 1: Table S3.

The mean RV insertion point T1 correlated significantly with the functional parameters mean PAP, mean right atrial pressure, and CI but not PVR, see Table 5 . There was also a significant correlation with mixed venous oxygen saturation ( $\mathrm{SvO} 2)$. LV free wall $\mathrm{T} 1$ correlated significantly with $\mathrm{CI}$ and $\mathrm{SvO} 2$, whilst septal T1 did not correlate with any of the RHC parameters. 
Table 3 Correlations between MR parameters in septal, RV insertion point and LV free wall T1

\begin{tabular}{llll}
\hline & Septal T1 (ms) & RV insertion point T1 (ms) & LV free wall T1 (ms) \\
\hline Age (years) & $r=-0.074 p=0.159$ & $r=0.007, p=1.000$ & $r=-0.086, p=0.168$ \\
BSA (m2) & $r=0.015, p=0.771$ & $r=0.041, p=0.444$ & $r=-0.006, p=0.915$ \\
Heart Rate (bpm) & $r=0.011, p=0.836$ & $r=-0.073, p=0.168$ & $r=-0.086, p=0.103$ \\
RVEDV Index (ml/m2) & $r=0.066, p=0.213$ & $r=0.265^{* *}, p<0.001^{* *}$ & $r=0.076, p=0.151$ \\
RVESV Index (ml/m2) & $r=0.027, p=0.615$ & $r=0.290, p<0.001^{* *}$ & $r=0.088, p=0.097$ \\
RVSV Index (\%) & $r=0.103, p=0.050$ & $r=0.003, p=0.958$ & $r=0.044, p=0.406$ \\
RVEF (\%) & $r=0.003, p=0.951$ & $r=-0.238, p<0.001^{* *}$ & $r=-0.080, p=0.129$ \\
LVEDV Index (ml/m2) & $r=-0.035, p=0.505$ & $r=-0.060, p=0.257$ & $r=-0.018, p=0.734$ \\
LVESV Index ml/m2) & $r=0.005, p=0.924$ & $r=0.011, p=0.841$ & $r=-0.051, p=0.335$ \\
LVSV Index (\%) & $r=0.051, p=0.335$ & $r=-0.066, p=0.217$ & $r=0.011, p=0.841$ \\
LVEF (\%) & $r=0.028, p=0.598$ & $r=0.238, p<0.001^{* *}$ & $r=0.050, p=0.347$ \\
Systolic septal angle $\left(^{\circ}\right)$ & $r=0.096, p=0.069$ & $r=0.253, p<0.001^{* *}$ & $R=0.017, p=0.742$ \\
Diastolic septal angle $\left(^{\circ}\right)$ & $r=0.157, p=0.003^{* *}$ & $r=0.229, p<0.001^{* *}$ & $r=0.017, p=0.742$ \\
RV mass index (g/m2) & $r=0.097, p=0.075$ & $r=0.072, p=0.173$ & $r=0.004, p=0.935$ \\
LV mass index (g/m2) & $r=-0.027, p=0.613$ & $r=0.180, p=0.001^{* *}$ & $r=0.041, p=0.435$ \\
VMl & $r=0.117, p=0.031^{*}$ & $r=-0.010, p=0.851$ \\
\hline
\end{tabular}

Legend: WHO world health organisation, IPAH idiopathic pulmonary arterial hypertension, CTD Connective tissue disease, CHD chronic heart disease, CTEPH chronic thromboembolic pulmonary hypertension, RVEDV right ventricular end diastolic volume, RVESV right ventricular end systolic volume, RVSV right ventricular stroke volume, RVEF right ventricular ejection fraction, VMI ventricular mass index, LVEDV left ventricular end diastolic volume, LVESV left ventricular end systolic volume, LVSV left ventricular stroke volume, LVEF left ventricular ejection fraction, VMI ventricular mass index

$P$ values indicate comparisons between patients with $\mathrm{PH}$ and the other two groups. ${ }^{*}$ signifies a $p$ value $<0.05,{ }^{* *}$ signifies a $p$ value $<0.025$

Patients with left heart disease showed significant correlations between RV insertion point $\mathrm{T} 1$ and mean PAP, mean right atrial pressure, and $\mathrm{SvO}$. Patients with left heart disease showed significant correlations with septal $\mathrm{T} 1$ and mPAP as well as SvO2. There were no correlations between LV free wall and right heart catheter parameters.

\section{Prognostic value of myocardial T1 in PAH}

Fifty-nine patients with PAH died during the follow up period of 29 months (standard deviation: 8 months), of which 43 had PAH.

Comparison of scaled Cox proportional hazard ratios are illustrated in Fig. 5. Univariate Cox proportional hazards regression analysis of patients with $\mathrm{PAH}$ showed that,

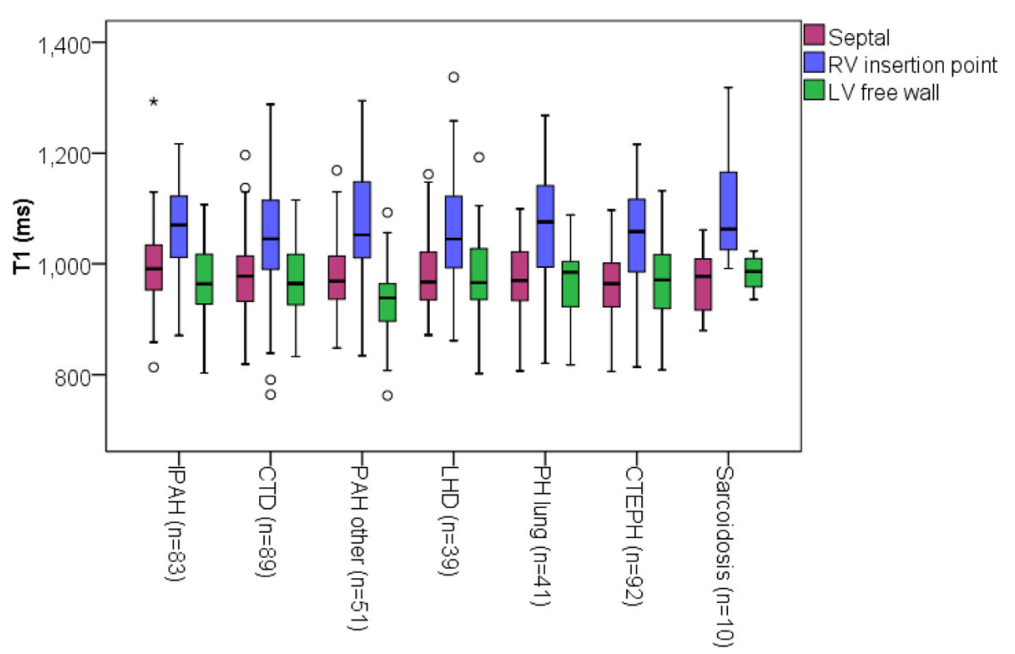

Fig. 4 Mean T1 in subgroups of patients with pulmonary hypertension. Box plot of mean T1 for septal, RV insertion point and LV free wall regions for pulmonary artery hypertension patient subtypes. PAH other includes all patients with PAH who do not have IPAH,CTD or CHD. O Represents outliers* represents far outliers 
Table 4 Linear regression analysis for RV insertion point T1

\begin{tabular}{|c|c|c|c|c|c|}
\hline & \multicolumn{2}{|c|}{$\begin{array}{l}\text { Unstandardized } \\
\text { Coefficients } \\
\end{array}$} & \multirow{2}{*}{$\begin{array}{l}\text { Standardized } \\
\text { Coefficients } \\
\text { Beta }\end{array}$} & \multirow[t]{2}{*}{$\mathrm{t}$} & \multirow[t]{2}{*}{$p$-value } \\
\hline & B & $\begin{array}{l}\text { Std. } \\
\text { Error }\end{array}$ & & & \\
\hline Constant & .845 & .073 & & 11.609 & $<0.001$ \\
\hline RVESVI & .000623 & 0.000247 & .209 & 2.524 & 0.008 \\
\hline RVEF & -0.000213 & 0.000500 & -.034 & -.425 & 0.921 \\
\hline VMI & .001 & .001 & .119 & 1.483 & 0.837 \\
\hline $\begin{array}{l}\text { Septal angle } \\
\text { systolic }\end{array}$ & .001 & .000 & .178 & 2.918 & 0.004 \\
\hline $\begin{array}{l}\text { Relative Area } \\
\text { Change }\end{array}$ & -.001 & .001 & -.066 & -.755 & 0.655 \\
\hline
\end{tabular}

Legend: RVESVI right ventricular end systolic index, RVEF right ventricular ejection fraction, VMI ventricular mass index

septal, LV free wall and RV insertion point T1 were not associated with mortality (septal: $p=0.522$; LV free wall: $p=0.258$; RV insertion point: $p=0.688$ ), see Table 6 .

\section{Reproducibility}

T1 values in the septum, RV insertion point and LV free wall were tested for absolute agreement with a random two-way model. The intraclass correlation coefficients for the interventricular septum, RV insertion points and LV free wall T1 values respectively were: $0.841,0.823$ and 0.792 respectively. The interclass correlation coefficients for the septum, RV insertion point and LV free wall $\mathrm{T} 1$ values respectively were: $0.844,0.742$ and 0.618 respectively. Reproducibility was found to be excellent in all regions in healthy subjects, intraclass correlation coefficients for the interventricular septum, RV insertion points and LV free wall T1 values were: 0.898, 0.934 and 0.852 respectively. The interclass correlation coefficients for the septum, RV insertion point and LV free wall T1 values were: $0.972,0.942$ and 0.824 respectively.

\section{Discussion}

Despite the clinical heterogeneity found in subtypes of $\mathrm{PAH}$ in the patients in this study, there were no significant differences found in myocardial T1 between the subtypes of this population. In particular, patients with left heart disease who were considered separately (due to a different aetiology and cardiac phenotype) were found to have no significant differences from patients with other forms of $\mathrm{PAH}$, this may be because the causal mechanism of $\mathrm{T} 1$ differences in PAH be present to some extent in all PAH subtypes. However, this may also be due to multiple casual mechanisms causing elevated T1 in PAHdue to the heterogeneity of the disease.

Patients with CTD have been previously found to have elevated T1 when compared to controls [22], however we did not find that patients with CTD had significantly different $\mathrm{T} 1$ in any region when compared with patients with idiopathic PAH. This indicates that any tissue changes caused by the presence of cardiac connective tissue were not detectable in this study, likely having a less significant effect than those of PAH itself, or patients with connective tissue disease did not have significant LV disease.

Two control groups were used to evaluate changes in the myocardium in patients with $\mathrm{PAH}$ : age matched healthy subjects and patients without PAH. Patients without PAH had significantly higher RV insertion point T1 when compared to healthy subjects. Although these patients do not meet the criteria for PAH diagnosis, they have a mean PAP of $19.4 \pm 2.7$, which is higher than seen in a healthy subject population. Additionally, T1 changes are not specific to $\mathrm{PAH}$, and therefore raised $\mathrm{T} 1$ in this group may be due to other disease such as connective tissue disease or left heart disease in this patient population.

Though both $R V$ insertion point $\mathrm{T} 1$ and $\Delta \mathrm{T} 1_{\mathrm{RS}}$ were successful at differentiating healthy subjects from patients with $\mathrm{PAH}$, they were not successful at differentiating control patients from patients with PAH. $\Delta \mathrm{T} 1_{\mathrm{RS}}$ was not significantly different between control groups, which suggests regional elevation of the insertion point $\mathrm{T} 1$ is characteristic of PAH rather than elevation of the whole myocardial T1. The lack of specificity of RV insertion

Table 5 Correlations between RHC parameters in septal, RV insertion point and LV free wall T1

\begin{tabular}{llll}
\hline RHC data & Septal T1 (ms) & RV insertion point T1 (ms) & LV free wall T1 (ms) \\
\hline Mean RAP $(\mathrm{mmHg})$ & $r=0.074, p=0.256$ & $r=0.160, p=0.011^{* *}$ & $r=0.025, p=0.706$ \\
Mean PAP $(\mathrm{mmHg})$ & $r=0.076, p=0.248$ & $r=0.165, p=0.011^{* *}$ & $r=0.025, p=0.706$ \\
PCWP $(\mathrm{mmHg})$ & $r=-0.002, p=0.982$ & $r=-0.053, p=0.434$ & $r=-0.032, r=0.634$ \\
PVR $\left(\right.$ dyne $\left./ \mathrm{s} / \mathrm{cm}^{3}\right)$ & $r=0.039, p=0.567$ & $r=0.099, p=0.145$ & $r=0.096, p=0.160$ \\
Cl $\left(\mathrm{I} / \mathrm{min} / \mathrm{m}^{2}\right)$ & $r=-0.003, p=0.964$ & $r=-0.079, p=0.234$ & $r=-0.166 . p=0.012^{* *}$ \\
SVO2 $(\%)$ & $r=-0.004, p=0.956$ & $r=-0.081, p=0.222$ & $r=-0.168, p=0.011^{* *}$ \\
SaO2 $(\%)$ & $r=0.119, p=0.070$ & $r=-0.065, p=0.322$ \\
\hline
\end{tabular}

Legend: $R A P$ right arterial pressure, $P A P$ pulmonary artery pressure, $P C W P$ pulmonary capillary wedge pressure, $P V R$ pulmonary vascular resistance, $C l$ cardiac index, $\mathrm{SvO} 2$ venous oxygen saturation, $\mathrm{SaO} 2$ arterial oxygen saturation

$P$ values indicate comparisons between patients with $\mathrm{PH}$ and the other two groups. ${ }^{*}$ signifies a $p$ value $<0.05,{ }^{* *}$ signifies a $p$ value $<0.025$ 


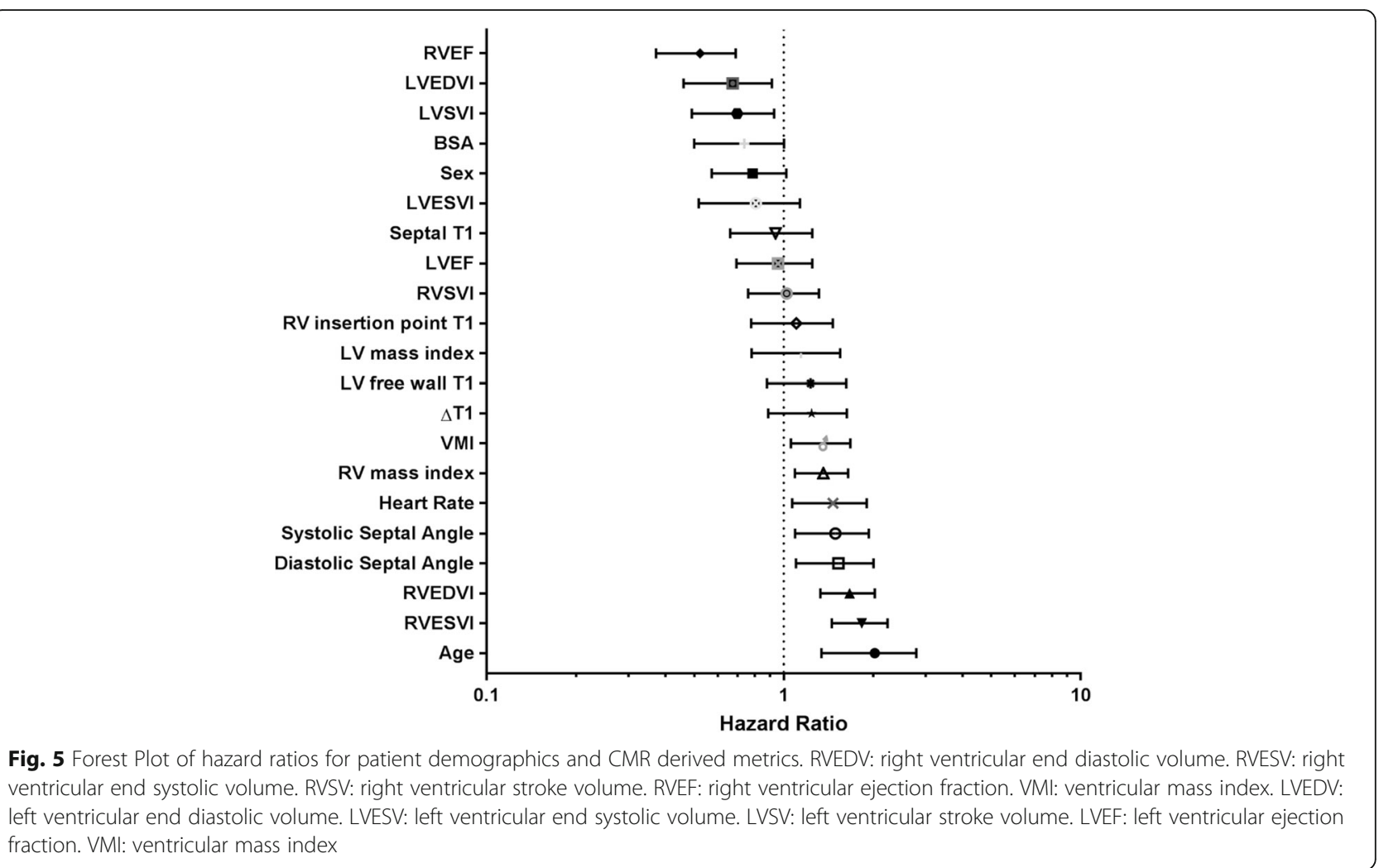

point $\mathrm{T} 1$ indicates that processes responsible for the elevated RV insertion point $\mathrm{T} 1$ are not solely confined to patients with $\mathrm{PAH}$.

The RV insertion points were the myocardial regions with the highest $\mathrm{T} 1$ and they correlated significantly with loss of RV function and RV remodelling. Systolic septal angle and RVESVI were independent predictors of $\mathrm{RV}$ insertion point $\mathrm{T} 1$, however these variables only predicted $12.4 \%$ of the variance seen in RV insertion point $\mathrm{T} 1$ in patients with $\mathrm{PAH}$. This may indicate that mechanisms which are not explicable solely through cardiac volume, mass and pressure changes are responsible for the tissue changes represented by $\mathrm{T} 1$.

Animal models of PAH with increased native T1 and LGE present in the RV insertion points have been shown to have increased interstitial collagen associated with fibre disarray, and increased connective tissue density [11]. It has been shown in two patients with $\mathrm{PAH}$ that fibrosis was present in the RV insertion point and it has been suggested that this is the mechanism

Table 6 Cox linear regression analysis for RV insertion point T1 in PAH. All variables have been normalised

\begin{tabular}{llll}
\hline & Univariate Hazard Ratio & Univariate 95\% confidence interval & Univariate P Value \\
\hline Age & 1.938 & $1.342-2.798$ & $<0.001^{* *}$ \\
Sex & 0.765 & $0.573-1.021$ & 0.077 \\
Septal T1 & 0.909 & $0.661-1.249$ & 0.552 \\
RV insertion point T1 & 1.067 & $0.778-1.464$ & 0.688 \\
LV free wall T1 & 1.195 & $0.879-1.624$ & 0.258 \\
$\Delta$ T1 $1_{\text {RS }}$ & 1.205 & $0.888-1.634$ & 0.237 \\
RVEDVI & 1.642 & $1.330-2.027$ & $<0.001^{* *}$ \\
RVESVI & 1.804 & $1.453-2.240$ & $<0.001^{* *}$ \\
RVEF & 0.506 & $0.372-0.690$ & $<0.001^{* *}$ \\
Systolic septal angle & 1.454 & $1.093-1.934$ & $0.011^{* *}$ \\
Diastolic septal angle & 1.485 & $1.099-2.008$ & $0.011^{* *}$ \\
RV mass Index & 1.341 & $1.091-1.649$ & $0.018^{* *}$
\end{tabular}

Legend: RVEDVI right ventricular end diastolic volume index, RVESVI right ventricular end systolic volume index, RVEF right ventricular ejection fraction 
driving LGE in PAH [23]. However, those patients also had an increase in interstitial space and a small increase of fat. A CMR case study of one patient with idiopoathic PAH showed myocardial disarray with increased collagen and fat between fibre bundles in the myocardium (plexiform fibrosis). That study suggests that the cause of LGE is not pathological, and instead arises from an exaggeration of the myocardial disarray and plexiform fibrosis where fibres from the RV and LV cross. It is suggested this exaggeration of myocardial disarray may arise from a combination of RV hypertrophy and increased shear forces upon the interventricular septum [24].

Interventricular septal angle is a measure of deviation of the LV septum due to the high pressure in the RV, which correlated strongly with RV insertion point T1 in patients with $\mathrm{PAH}$ and was found to be an independent linear predictor of RV insertion point T1. Even in patients with left heart disease, who do not exhibit divergence of the LV septum to the same degree as other patients with $\mathrm{PAH}, \Delta \mathrm{T} 1_{\mathrm{RS}}$ correlated significantly with septal angle. The T1 value of the LV free wall, which would not experience stress/strain due to elevated ventricular pressure, was found to show no correlation between T1 and septal angle and was not elevated in patients with PAH when compared to patients without $\mathrm{PAH}$. Therefore, our data suggests that the regional elevated native $\mathrm{T} 1$ in the insertion points is significantly related to the displacement of the interventricular septum in PAH. This supports the suggestions from histological studies that changes in myocardial $\mathrm{T} 1$ and the presence of LGE may be due to exaggerated fibre disarray in the RV insertion points of the myocardium due to stress on the septum and RV insertion points in PAH.

RV insertion point $\mathrm{T} 1$ was not an independent significant predictor of mortality in patients with PAH. Although LGE and elevated native $\mathrm{T} 1$ has been observed in the interventricular septum in PAH [3-5], elevated native T1 was not observed in this study. This may be because regions of interest were placed in the middle of the interventricular septum, whereas LGE imaging shows myocardial changes extending from the insertion points and therefore weakest enhancement at the central region of the septum.

Reproducibility data was found to be in line with that previously observed in patient T1 mapping studies [24]. The RV insertion points were found to be less reproducible than the interventricular septum. This may be due to higher $\mathrm{T} 1$ heterogeneity in this region leading to greater $\mathrm{T} 1$ variation when region of interest placement differs. T1 values tended to be less reproducible in patients with PAH than healthy subjects, which is consistent with other studies findings [24]. It may be that relatively poor breath-holding of the patient cohort contributes a greater degree of motion artefact resulting in weaker reproducibility.
Myocardial T1 did not provide additive diagnostic or prognostic information in this PAH cohort. It may be that myocardial changes are a secondary effect of cardiac remodelling and are therefore less relevant in this disease. However, it may also be that traditional region of interest based analysis of T1 is less suitable for this patient population due to heterogeneous $\mathrm{T} 1$ across the myocardium resulting in weaker reproducibility.

In conclusion, the RV insertion points have been shown to be the region where $\mathrm{T} 1$ changes are most significantly related to cardiac function in PAH. Myocardial T1 did not differ significantly between PAH subtypes including LHD. The T1 in the RV insertion point was significantly associated with RV volume and function in the patients scanned. Interventricular septal angle independently predicted RV insertion point $\mathrm{T} 1$ in patients with PAH suggesting that regional elevation in the RV insertion point may be linked to increased stress on the interventricular septal wall. T1 in patients with PAH was not found to be prognostic independent of RV size and function. Overall, T1 mapping does not appear to predict the subtype of $\mathrm{PAH}$, or provide additive value over $\mathrm{RV}$ volume, mass and geometry in the prognostic evaluation of patients with PAH.

\section{Limitations}

It is a limitation of this study that concurrent right heart catheter and CMR data were not present for all subjects. The follow up period for this study was relatively short, with $85 \%$ of patients alive at the follow-up date. Therefore, census at a later point may be required to provide more definitive evidence of whether RV insertion point T1 has independent prognostic value. Further work to evaluate regions of interest at the inferior and superior portions of the interventricular septum may clarify whether septal changes are present in these regions. The relationship between $\mathrm{T} 1$ and LGE in the RV insertion points was not evaluated here and further work may be warranted to evaluate this thoroughly in patients with PAH.

\section{Additional file}

Additional file 1 Table S1. Subject characteristics for pulmonary artery sub groups. Table S2. Pulmonary artery hypertension subject. Table S3. Right heart catheterization characteristics of subjects. (DOCX $25 \mathrm{~kb}$ )

\section{Abbreviations}

BSA: Body surface area; bSSFP: Balanced steady state free precession; CHD: Congenital heart diseases; Cl: Cardiac index; CMR: Cardiovascular magnetic resonance; CTD: Connective tissue disease; CTEPH: Chronic thromboembolic pulmonary hypertension; ECG: Electrocardiogram; IPAH: Idiopathic pulmonary artery hypertension; LGE: Late gadolinium enhancement; LV: Left ventricle/left ventricular; LVEDV: Left ventricular enddiastolic volume; LVEF: Left ventricular ejection fraction; LVESV: Left 
ventricular end-systolic volume; MOLLI: Modified Look-Locker inversion recovery; PAH: Pulmonary artery hypertension; PAP: Pulmonary artery pressure; PCWP: Pulmonary capillary wedge pressure; PVR: Pulmonary vascular resistance; PVRI: Pulmonary vascular resistance index; RAP: Right atrial pressure; RV: Right ventricle/right ventricular; RVEDV: Right ventric ular enddiastolic volume; RVEF: Right ventricular ejection fraction; RVESV: Right ventricular end-systolic volume; SvO2: Mixed venous oxygen saturation; VMI: Ventricular mass index

\section{Acknowledgments}

None.

\section{Availability of data and material}

All data generated or analysed during this study are included in this published article and its supplementary information files.

\section{Funding}

This work was supported by NIHR grant NIHR-RP-R3-12-027, MRC grant MR/ M008894/1 and the Wellcome Trust 205188/Z/16/Z.

\section{Authors contributions}

LCS wrote the manuscript, implemented the imaging methods and analysis, validated the MOLLI sequence, wrote the code for T1 analysis and undertook statistical analysis. CJ provided clinical insight into pulmonary vascular disease and assisted with statistical analysis. NS implemented the imaging methods and validated the MOLLI sequence and analysis. DC and $\mathrm{CH}$ undertook the MR imaging of patients and healthy subjects. JW supervised technical implementation of the imaging methods and analysis, supervised the study design and critically revised the manuscript. AS supervised the study design and designed the clinical hypothesis and critically revised the manuscript. DK, CE and RC provided clinical insight into pulmonary vascular disease and mechanisms of $\mathrm{T} 1$ and helped draft the manuscript. MG provided imaging methods development and drafting the manuscript. VP provided insight into the role of $\mathrm{T} 1$ in the study design and drafting the manuscript. All authors read and approved the final manuscript.

\section{Ethics approval and consent to participate}

Approval for analysis of imaging data was granted by the local research ethics committee and consent was waived for this retrospective database study.

\section{Competing interests}

The authors declare that they have no competing interests.

\section{Publisher's Note}

Springer Nature remains neutral with regard to jurisdictional claims in published maps and institutional affiliations.

\section{Author details}

${ }^{1}$ POLARIS, Academic Radiology, Department of Infection, Immunity and Cardiovascular Disease, University of Sheffield, Sheffield, UK. ²Division of Bioengineering and Bioinformatics, Graduate School of Information Science and Technology, Hokkaido University, Hokudai, Japan. ${ }^{3}$ Institute for Experimental and Translational Cardio Vascular Imaging, University Hospital Frankfurt, Frankfurt, Germany. ${ }^{4}$ Pulmonary Vascular Disease Unit, Sheffield Teaching Hospitals NHS Trust, Sheffield, UK. ${ }^{5}$ INSIGNEO, Institute for in-silico medicine, Sheffield, UK. ${ }^{6}$ University of Cambridge School of Clinical Medicine, Cambridge University, Cambridge, UK.

Received: 26 April 2018 Accepted: 24 October 2018 Published online: 03 December 2018

\section{References}

1. Puntmann VO, Isted A, Hinojar R, Foote L, Carr-White G, Nagel E. T1 and T2 mapping in recognition of early cardiac involvement in systemic sarcoidosis. Radiology. 2017;285(1):63-72.

2. Tahir $\mathrm{E}$, Sinn M, Bohnen S, et al. Acute versus chronic myocardial infarction: diagnostic accuracy of quantitative native $\mathrm{T} 1$ and $\mathrm{T} 2$ mapping versus assessment of edema on standard T2-weighted cardiovascular MR images for differentiation. Radiology. 2017;285(1):83-91.
3. Roller FC, Wiedenroth C, Breithecker A, et al. Native T1 mapping and extracellular volume fraction measurement for assessment of right ventricular insertion point and septal fibrosis in chronic thromboembolic pulmonary hypertension. Eur Radiol. 2016.

4. Spruijt OA, Vissers L, Bogaard HJ, Hofman MB, Vonk-Noordegraaf A, Marcus JT. Increased native T1-values at the interventricular insertion regions in precapillary pulmonary hypertension. Int J Cardiovasc Imaging. 2016;32(3):451-9.

5. Reiter U, Reiter G, Kovacs G, et al. Native myocardial T1 mapping in pulmonary hypertension: correlations with cardiac function and hemodynamics. Eur Radiol. 2017;27(1):157-66.

6. Mehta BB, Auger DA, Gonzalez JA, et al. Detection of elevated right ventricular extracellular volume in pulmonary hypertension using accelerated and navigator-gated look-locker imaging for cardiac T1 estimation (ANGIE) cardiovascular magnetic resonance. J Cardiovasc Magn Reson. 2015;17:110.

7. Pagano JJ, Chow K, Khan A, et al. Reduced right ventricular native myocardial $\mathrm{T} 1$ in Anderson-Fabry disease: comparison to pulmonary hypertension and healthy controls. PLOS ONE [electronic Resource]. 2016;11(6):e0157565.

8. Freed BH, Gomberg-Maitland M, Chandra S, et al. Late gadolinium enhancement cardiovascular magnetic resonance predicts clinical worsening in patients with pulmonary hypertension. J Cardiovasc Magn Reson. 2012;14:11.

9. Swift AJ, Rajaram S, Campbell MJ, et al. Prognostic value of cardiovascular magnetic resonance imaging measurements corrected for age and sex in idiopathic pulmonary arterial hypertension. Circ Cardiovasc Imaging. 2014; 7(1):100-6.

10. Swift AJ, Rajaram S, Capener D, et al. LGE patterns in pulmonary hypertension do not impact overall mortality. J Am Coll Cardiol Img. 2014;7(12):1209-17.

11. Garcia-Alvarez A, Garcia-Lunar I, Pereda D, et al. Association of myocardial T1-mapping CMR with hemodynamics and RV performance in pulmonary hypertension. J Am Coll Cardiol Img. 2015;8(1):76-82.

12. van Wolferen SA, Marcus JT, Boonstra A, et al. Prognostic value of right ventricular mass, volume, and function in idiopathic pulmonary arterial hypertension. Eur Heart J. 2007;28(10):1250-7.

13. D'Alonzo GE, Barst RJ, Ayres SM, et al. Survival in patients with primary pulmonary hypertension. Results from a national prospective registry. Ann Intern Med. 1991;115(5):343-9.

14. Vanderpool RR, Pinsky MR, Naeije R, et al. RV-pulmonary arterial coupling predicts outcome in patients referred for pulmonary hypertension. Heart. 2015;101(1):37-43.

15. van de Veerdonk MC, Kind T, Marcus JT, et al. Progressive right ventricular dysfunction in patients with pulmonary arterial hypertension responding to therapy. J Am Coll Cardiol. 2011;58(24):2511-9.

16. Dawes TJW, de Marvao A, Shi W, et al. Machine learning of three-dimensional right ventricular motion enables outcome prediction in pulmonary hypertension: a cardiac MR imaging study. Radiology. 2017;283(2):381-90.

17. Hurdman J, Condliffe R, Elliot CA, et al. ASPIRE registry: assessing the Spectrum of pulmonary hypertension identified at a REferral Centre. Eur Respir J. 2012:39(4):945-55.

18. Swift AJ, Rajaram S, Hurdman J, et al. Noninvasive estimation of PA pressure, flow, and resistance with CMR imaging: derivation and prospective validation study from the ASPIRE registry. JACC Cardiovasc Imaging. 2013;6(10):1036-47.

19. Barber DC, Hose DR. Automatic segmentation of medical images using image registration: diagnostic and simulation applications. J Med Eng Technol. 2005;29(2):53-63.

20. Xue H, Shah S, Greiser A, et al. Motion correction for myocardial T1 mapping using image registration with synthetic image estimation. Magn Reson Med. 2012;67(6):1644-55.

21. Rogers T, Dabir D, Mahmoud I, et al. Standardization of T1 measurements with MOLLI in differentiation between health and disease--the ConSept study. J Cardiovasc Magn Reson. 2013;15:78.

22. Mayr A, Kitterer D, Latus J, et al. Evaluation of myocardial involvement in patients with connective tissue disorders: a multi-parametric cardiovascular magnetic resonance study. J Cardiovasc Magn Reson. 2016;18:67.

23. McCann GP, Gan CT, Beek AM, Niessen HW, Vonk Noordegraaf A, van Rossum AC. Extent of MRI delayed enhancement of myocardial mass is related to right ventricular dysfunction in pulmonary artery hypertension. AJR Am J Roentgenol. 2007;188(2):349-55.

24. Bradlow WM, Assomull R, Kilner PJ, Gibbs JS, Sheppard MN, Mohiaddin RH. Understanding late gadolinium enhancement in pulmonary hypertension. Circ Cardiovasc Imaging. 2010;3(4):501-3. 